\title{
THE INTERRELATIONSHIPS OF HORMONES, FAT'TY ACID AND GLUCOSE IN THE PROVISION OF ENERGY
}

\author{
P. J. Randle, M.A., Ph.D., M.B., M.R.C.P. \\ Department of Biochemistry, University of Bristol.
}

AN important and characteristic property of living matter is its ability to transfer energy released from the breakdown of foodstuffs to other energy-requiring processes such as the synthesis of cell materials or the performance of work. The energy transfer is effected by chemical substances chief amongst which are nucleoside triphosphates such as ATP (adenosine triphosphate) and for reductive syntheses (such as fatty acid or steroid synthesis) reduced coenzymes (abbreviated as $\mathrm{NADH}_{2}$ or $\mathrm{NADPH}_{2}$ which are reduced coenzyme I and II respectively). In mammalian cells ATP and reduced coenzymes are generated for the most part by respiration and the major fuels for respiration are glucose and fatty acids and ketone bodies. These reactions, concerned with the formation and utilisation of ATP and reduced coenzymes, constitute what is known as energy metabolism in cells. Their control necessarily plays an important part in the overall regulation of cell metabolism. This chapter is concerned largely with the importance of the control of energy metabolism in mammalian muscle to the actions of hormones and to the metabolic disturbances of diabetes and other endocrine disorders. The discussion will be confined to the ways in which cells increase or decrease the production of ATP and reduced coenzymes according to needs and with the ways in which cell metabolism is adjusted by the availability of glucose, fatty acids and oxygen. Together these processes comprise what is known collectively as respiratory control. To discuss the reactions involved in the use of ATP and reduced coenzymes is beyond the scope of this chapter but it may be noted that one or both participate in the synthesis of all major cell constituents (nucleic acids, proteins, lipids, carbohydrates and coenzymes) and in such major physiological processes as muscular contraction, the passage of nerve impulses and the movements of ions, amino acids and sugars across cell membranes against concentration gradients.

Respiratory control in muscle comprises three distinct but related processes. The first is concerned with the control of oxygen consumption. In healthy cells the consumption of oxygen is presumably controlled by the rate of ATP utilisation and mediated by the concentration of ADP (adenosine diphosphate) formed in the utilisation of ATP (Chance, 1959). The second type of respiratory control is concerned with the regulation of rates of glycolysis and glucose uptake by the intracellular concentration of ATP. This type of control was first observed by Pasteur who noted that glycolysis in yeast is greater in the absence of oxygen than in its presence. It is now recognised that the Pasteur effect (the inhibition of glycolysis by respiration) is exhibited by most cells and tissues including muscle (Randle, 1956; Morgan, Randle and Regen, 1959). It has been attributed to the fact that anaerobic metabolism of glucose yields less ATP than aerobic metabolism (Randle and Smith, 1958a). In keeping with this it was observed that drugs such as salicylate or 2:4-dinitrophenol (which interfere with the synthesis of ATP by respiration) accelerate glucose uptake and glycolysis. As will be seen later the reactions involved in the control of glycolysis may be directly controlled by the intracellular concentration of ATP and of its breakdown products 5'AMP (adenosine mono-phosphate) and $\mathrm{Pi}$ (inorganic phosphate). The third type of respiratory control is concerned with the interactions of glucose and fatty acid metabolism. In muscle the provision of fatty acids or ketone bodies for respiration can impair the oxidation of glucose (Drury and Wick, 1953) and diminish glucose uptake and glycolysis (Williamson and Krebs, 1961; Shipp, Opie and Challoner, 1961; Newsholme, Randle and Manchester, 1962; Garland, Newsholme \& Randle, 1962). As will be discussed later this control is exercised through changes in the intracellular concentrations of coenzyme A, acetyl CoA and citrate (intermediates in the oxidation of glucose, fatty acids and ketone bodies). The metabolism of glucose by glycolysis can also diminish the oxidation of fatty acids in muscle by facilitating their incorporation into tri- 


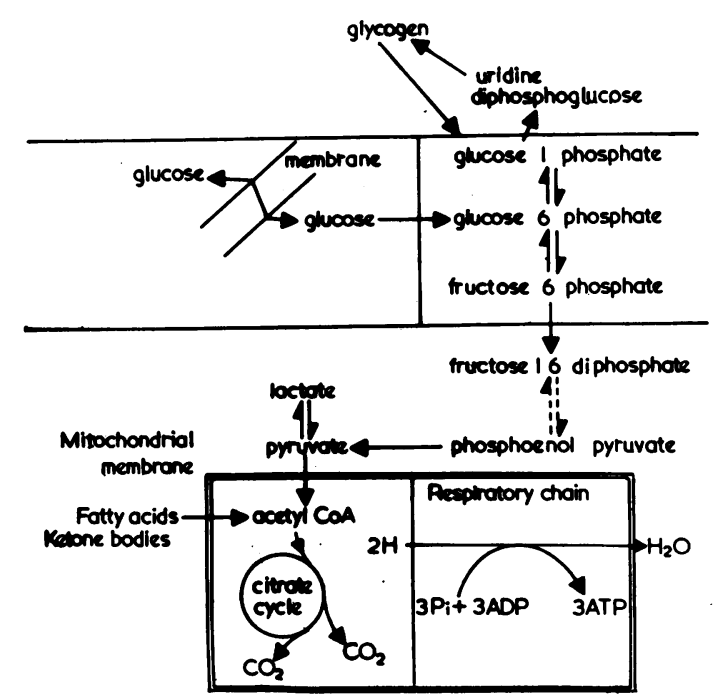

Fig. 1.-Glucose, fatty acid and energy metabolism in muscle. Upper panel; glycogen synthesis via UDP glucose and UDP glucose glycogen glucosyl transferase and glycogen breakdown catalysed by phosphorylase; Middle panel; on right hexose monophosphate pool and on left reactions of glucose uptake (membrane transport and phosphorylation of glucose catalysed by hexokinase); Lower panel; outside of the mitochondrion the reactions of glycolysis commencing with the formation of fructose 1:6-diphosphate catalysed by phosphofructokinase and ending with the formation of pyruvate catalysed by pyruvate kinase; inside of the mitochondrion the substrate respiratory pathways leading to the oxidation of pyruvate and fatty acids and ketone bodies, and the reactions of the respiratory chain and of respiratory chain phosphorylation. For further explanation see text.

glyceride (Garland and Randle, 1963). This third type of respiratory control is of particular importance in diabetes because of the likelihood that in some cases the impaired glucose tolerance is secondary to an abnormally high rate of release of fatty acids for respiration.

Before considering the general nature of control of cell metabolism and the mechanisms of respiratory control and their importance it may be useful to summarise briefly the reactions involved in glucose and fatty acid metabolism and in respiration.

\section{Energy metabolism in muscle}

The reactions involved in the metabolism of glucose by glycolysis and oxidation; in the oxidation of fatty acids and ketone bodies; and in the associated processes of fatty acid storage and release are briefly summarised in Fig. 1 and Fig. 2. It is important to note that the metabolism of glucose by glycolysis to pyruvate

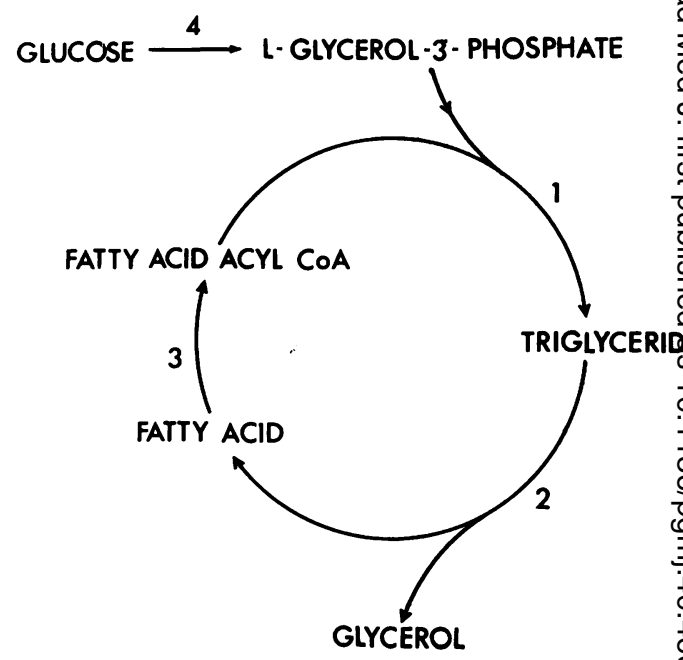

FIG. 2.-Triglyceride synthesis and breakdown.

1. Esterification of fatty acid acyl CoA triglyceride.

2. Lipolysis catalysed by esterases.

3. Conversion of fatty acids to acyl CoA de rivatives. 4. Formation of glycerolphosphate by glycol
sis.

necessarily precedes the respiratory proce in which pyruvate is oxidised. This is impore tant because glycolysis can proceed in the absence of oxygen. This is because the onlo oxidative reaction in glycolysis (the oxidatio of glyceraldehyde phosphate) is coupled to the reduction of pyruvate to lactate and is theres fore independent of molecular oxygen.

Respiration, whether of glucose or fatt acids or ketone bodies, involves two separate but distinct processes. These are the substrate pathways in which enzyme-catalysed reactions in sequence lead to their conversion to carbog dioxide and hydrogen. The latter is either transferred to NAD or NADP or discharged as $\mathrm{H}+$ with the transfer of electrons to the respiratory chain. The second pathway is the respiratory chain coupled with the associate process of respiratory chain phosphorylation. In this pathway electrons are transferred from hydrogen to oxygen to form water and ADR and $\mathbf{P i}$ are reacted to form ATP. It is through respiratory chain phosphorylation that the greater part of ATP is synthesised under aerobic conditions. The oxidation of each pait of hydrogen atoms from $\mathrm{NADH}_{2}$ or NADPH may lead to the synthesis of three molecules of ATP (i.e. to a $P: O$ ratio of 3). In the oxidation of glucose the total yield of ATP mole is 32 of which 28 are derived from respir 
atory chain phosphorylation, 2 from substrate flow in the citrate cycle and 2 from glycolysis. In the oxidation of the fatty acid palmitate the total yield of ATP/mole is 134, of which 126 are derived from respiratory chain phosphorylation.

The reactions involved in glycolysis and in the oxidation of glucose are shown in Fig. 1. They may conveniently be grouped for discussion of metabolism control into a series of discrete processes interconnected by essentially irreversible reactions. The hexose monophosphate pool is the name given to three compounds: glucose 1-phosphate, glucose 6phosphate and fructose 6-phosphate, whose interconversion is catalysed by two enzymes, phosphoglucomutase and phosphoglucoisomerase. These reactions are reversible so that the compounds in this pool are interconvertible. Connected with the pool are the processes of glucose uptake, glycolysis and the glycogen cycle. Glucose uptake involves in sequence membrane transport of glucose followed by phosphorylation of the sugar within the cell to glucose 6-phosphate catalysed by hexokinase. The former is reversible, the latter not and the net flow of glucose is into the cell. The glycogen cycle involves independent pathways of synthesis (through the intermediate formation of UDP glucose and transfer of glucose from the latter to glycogen catalysed by UDP glucose glycogen glucosyl transferase) and of breakdown (catalysed by phosphorylase). These reactions are essentially irreversible in the cell. Glycolysis involves phosphorylation of fructose 6-phosphate to fructose 1:6disphosphate (catalysed by phosphofructokinase) followed by a chain of reactions leading to the formation of pyruvate. Two of the reactions are essentially irreversible, namely the phosphofructokinase reaction (the first) and the pyruvate kinase reaction (the last in the sequence). Reversal of glycolysis involves additional enzymes which are present in liver but not in muscle. The oxidation of pyruvate proceeds by an irreversible reaction catalysed by pyruvate dehydrogenase and leading through reaction with $\mathrm{CoA}$ (coenzyme A) to the formation of acetyl CoA. The latter is then oxidised by the reaction of the citrate cycle and of the respiratory chain to carbon dioxide and water. The oxidation of fatty acids and ketone bodies proceeds by reaction with $\mathrm{CoA}$ to form acyl CoA derivatives foilowed by oxidation and cleavage to acetyl CoA. The latter is then oxidised through the citrate cycle. It should be noted that the oxidation of pyruvate, fatty acids and ketone bodies proceeds through a common intermediate (acetyl CoA) and that $\mathrm{CoA}$ is an essential participant in the oxidation of each. The components of the respiratory chain and of respiratory chain phosphorylation and the enzymes involved in the oxidation of fatty acids, ketone bodies and pyruvate are located in mitochondria. The remaining reactions involved in glucose metabolism are located in the cytoplasm.

The reactions involved in the storage and release of fatty acids are shown in Fig. 2. These reactions are common both to adipose tissue and muscle though the store of triglyceride is much more substantial in adipose tissue. It should be noted that fatty acids released by breakdown of adipose tissue glycerides can be transferred to muscle through the bloodstream in combination with albumin (plasma non-esterified fatty acids or NEFA). The breakdown of triglyceride involves hydrolysis by esterases to glycerol and fatty acid (lipolysis). The fatty acid is then converted to the acyl CoA derivative which may either be oxidised or reconverted to triglyceride by reaction with glycerol phosphate. In adipose tissue (but not in muscle) fatty acids may be additionally released to plasma albumin. It should be noted that glycerol is not metabolised in adipose tissue or muscle and that the glycerol phosphate required for triglyceride synthesis is derived from glucose (by glycolysis).

\section{Control of glucose and fatty acid metabolism}

\section{Fundamental considerations.}

The control of metabolism in cells involves control of rates of flow in metabolic pathways. This is invariably achieved by controlling the rate of a limited number (usually one) of the enzyme-catalysed reactions or transport processes. These rate-limiting reactions may be described as the "checkpoints" in metabolism. With few exceptions these checkpoints are irreversible reactions. In the pathways discussed previously the checkpoints are, for glucose uptake, either membrane transport or phosphorylation of glucose; for glycogen breakdown the phosphorylase reaction; for glycogen synthesis the UDPG glycogen glucosyl trans ferase reaction; for glycolysis the phosphofructokinase reaction; and for pyruvate oxidation the pyruvate dehydrogenase reaction. The rate of fatty acid oxidation may be controlled in part by the rate of release of fatty acids from triglyceride stores and in part by the availability of NAD (as opposed to $\mathrm{NADH}_{2}$ ) in the mitochondrion. The rate of 
lipolysis is controlled by the activity of an esterase (Vaughan, Berger \& Steinberg, 1964). The checkpoint in triglyceride synthesis is not known.

The control of enzyme-catalysed reactions of transport processes may be summarised briefly as follows:

1. Genotropic. This involves the control of the synthesis of enzymes in cells or of transport systems in cell membranes by the genes (deoxyribonucleic acid). The synthesis of these proteins occurs largely in ribosomes attached to the endoplasmic reticulum in the cell cytoplasm and the ribosomes are activáted to synthesise particular proteins by m-RNA (messenger RNA) which transmits genetic information about the structure of the protein to be made from the genes in the nuclei. According to concepts developed by Monod, Changeux and Jacob (1963) the synthesis of m-RNA may be controlled by a segment of DNA containing operator genes (an operon) which it is postulated are controlled by a repressor protein synthesised under the activity of a regulator gene. The action of a repressor would prevent synthesis of particular enzyme proteins and would be modified by combination with particular cell metabolites which convey information about the need for synthesis of a particular protein. It seems possible that some hormones which induce the synthesis of particular proteins (e.g. cortisol) may act by combination with a particular repressor. Evidence for genotropic control of carbohydrate or fat metabolism in muscle is at present lacking. In the liver on the other hand there is evidence for genotropic control of glucokinase; the enzyme largely disappears from the liver in diabetes or following starvation in the rat and reappears following treatment with insulin or refeeding (Vinuela, Salas and Sols, 1963; Walker and Rao, 1964).

2. Enzymotropic. Three types of control of the activity of enzyme proteins are recognised. These are: -

(a) control by substrate availability particularly where enzyme is separated from substrate by a membrane. Examples of this type are control of the access of glucose to hexokinase in muscle by the rate of transport of glucose across the cell membrane, and the control of oxygen consumption by the availability of ADP to the respiratory chain (see above).

(b) control of enzyme activity by the action of common metabolites functioning as activators or inhibitors of enzymes. This type of control has been named allosteric by Monod and others (1963) and they have concluded thă enzymes showing this have in addition to the active site (at which the reaction is catalysed) an allosteric site at which inhibitors or activators exert their effects. Examples of this type $\vec{g}$ control are inhibition of hexokinase by glucost 6-phosphate; activation of UDP gluco glycogen glucosyl transferase by glucose $\sigma_{\text {; }}$ phosphate; activation of phosphorylase " $b$ " 而 muscle by $5^{\prime}$ AMP and its inhibition by ATP. inhibition of phosphofructokinase by ATP and citrate and its activation by $5^{\prime} \mathrm{AMP}$ and $\mathrm{Pi}$. $\overrightarrow{0}$ (c) control of enzyme activity by phosphory lation or dephosphorylation of the enzymes protein catalysed by other regulator enzymes. Examples of this are the activation of liver phosphorylase by phosphorylation of the enzyme (controlled by glucagon and adrenas line) and activation of muscle UDP glucoses glycogen glucosyl transferase by dephosphorys lation of the enzyme (controlled by insulinf) (see Sutherland and Rall, 1960; Larner 1964و

\section{Mechanism of respiratory control \\ Control of oxygen uptake}

According to Chance (1959) oxygen uptake may be controlled by the availability of ADP as an acceptor for phosphate in respirato chain phosphorylation (because of the coup of this phosphorylation with the respiratery chain). It follows from this concept that oxygen consumption may only be stimulate when ATP utilisation (which generates ADP is accelerated or when phosporylation an respiration are uncoupled. The latter may $b \overrightarrow{\vec{\epsilon}}$ achieved by drugs such as salicylate or $2: 43$ dinitrophenol or by excessive amounts of thyroxine as in thyrotoxicosis. Physiologica? concentrations of thyroxine do not uncouple phosphorylation from respiration (Tata, Ernster. Lindberg, Arrhenius, Padercen and Hedman. 1963) and the rise in oxygen consumption. induced in hypothyroid patients by therapeutie doses may thus be due to stimulation of ATP utilisation e.g. by a general increase in activity and by stimulation of protein synthesis.

Control of glucose metabolism by ATP supply

In an earlier section it was pointed out that agents such as anoxia or drugs such a\$ salicylate or 2:4-dinitrophenol which inhibif ATP formation accelerate glucose uptake glycolysis and glycogen breakdown in muscle The reactions involved in this accelerations are glucose transport (Randle and Smith: 1958b; Morgan and others, 1959); glucose phosphorylation (Morgan and others, 1959) phosphofructokinase (Newsholme and Randle $e_{\mathbb{D}}^{?}$ 
1961); and phosphorylase (Cornblath, Randle, Parmeggiani and Morgan, 1963). The mechanisms so far as they are known involve allosteric interactions with ATP and $5^{\prime}$ AMP and Pi, since the actions of these agents lower the intracellular concentration of ATP and raise those of $5^{\prime}$ AMP and Pi. Phosphofructokinase is inhibited by ATP and activated by $5^{\prime}$ AMP and Pi (Passonneau and Lowry, 1962); phosphorylase " $b$ " in muscle is activated by 5 ' AMP and this activation is blocked by ATP (Parmeggiani and Morgan, 1962). The activation of hexokinase may involve a fall in the intracellular concentration of glucose 6-phosphate (an inhibitor of the enzyme) and a rise in $\mathrm{Pi}$ (an activator-Tiederman and Born, 1959). The control of glucose transport has yet to be elucidated. It is important to note that hormones which stimulate ATP utilisation will necessarily accelerate glucose uptake, glycolysis and glycogen breakdown unless their action also leads to the provision of sufficient fatty acid to support respiration. It should be noted also that hormones which uncouple phosphorylation from respiration will also accelerate glucose uptake, glycolysis and glycogen breakdown. This may be seen with toxic concentrations of thyroxine as in hyperthyroidism.

\section{Interactions of glucose and fatty acid metabolism}

Evidence has recently been summarised to show that there is a reciprocal relationship between the utilisation of glucose and fatty acid in muscle. This relationship has been named the Glucose Fatty Acid Cycle (Randle, Garland, Hales and Newsholme, 1963). In rat heart or diaphragm muscle perfused or incubated in vitro with medium containing fatty acids or ketone bodies, glucose uptake, glycolysis and glucose oxidation are impaired. These changes are due to inhibitory effects of respiration of these substrates on glucose transport, glucose phosphorylation, the phosphofructokinase reaction and the pyruvate dehydrogenase reaction (Newsholme and others, 1962; Garland and others, 1962; Randle and others, 1963). The fundamental mechanism underlying these changes appears to be an increase in the ratio of the concentration of acetyl $\operatorname{CoA}$ to that of $\mathrm{CoA}$ which inhibits pyruvate dehydrogenase (Garland and Randle, 1964); and a rise in the intracellular concentration of citrate which inhibits phosphofructokinase, perhaps by allosteric interaction (Garland, Randle and Newsholme, 1963; Parmeggiani and Bowman, 1963). The mechanism of the altered rates of glucose transport and phosphorylation are not yet known though the latter may be due to an increase in the intracellular concentration of glucose 6-phosphate, an inhibitor of hexokinase (Newsholme and others, 1962). The reciprocal relationship between the metabolism of glucose and of fatty acids arises because of the inhibitory effects of glucose metabolism on the release of fatty acids for oxidation from either muscle glycerides or adipose tissue glycerides. This has been shown by in vitro experiments in which addition of glucose to the incubation medium lowers the intracellular concentration of fatty acids in rat heart and diaphragm muscles (Garland and Randle, 1963) and inhibits the release of fatty acids from adipose tissue. It has also been shown in vivo in man and other animals where the administration of glucose lowers the plasma concentrations of nonesterified fatty acids.

\section{The Glucose Fatty Acid Cycle and Metabolic Disturbances of Diabetes Mellitus}

In normal animals the smooth functioning of the Glucose Fatty Acid Cycle allows for continuous readjustment of the relative rates of utilisation of glucose and fatty acids as fuels for respiration in muscle and other tissues. The hormones largely concerned with this regulation are insulin, which reduces the rate of release of fatty acids and promotes the uptake of glucose and (by inhibiting release of fatty acids for oxidation) the oxidation of glucose, and growth hormone and corticosteroids which together may promote the release of fatty acids for oxidation and thereby inhibit the oxidation of glucose. After each intake of carbohydrate the release of fatty acids declines and the oxidation of glucose is enhanced. Between periods of carbohydrate intake the release of fatty acids is accelerated and the uptake and oxidation of glucose is diminished. These changes are assisted by the release of insulin after carbohydrate intake and possibly by the release of growth hormone during periods of carbohydrate deprivation. In alloxan-diabetes in the rat the normal relationship between glucose and fatty acid metabolism is disturbed. In the muscles of alloxan-diabetic rats in vitro the action of insulin on the disposal of glucose is impaired and the rates of glucose transport and phosphorylation and of the phosphofructokinase and pyruvate dehydrogenase reactions are diminished. These changes are very similar to those which have been induced in normal muscle by the provision of fatty acids and direct evidence for increased provision of fatty acids for respiration by accelerated breakdown 
of muscle glycerides in diabetic muscle has been obtained (Randle and others, 1963; Garland and Randle, 1963; Garland and others, 1963). The defects in glucose metabolism in muscles of diabetic rats may be corrected either by treatment of the rat with insulin or by hypophysectomy and each of these treatments corrects the abnormally high rate of release of fatty acids. These findings suggest that those defects in glucose metabolism in alloxan-diabetes which lead to insensitivity towards actions of insulin on glucose disposal in muscle are secondary manifestations of augmented release and oxidation of fatty acids.

In human diabetes the earliest manifestations are those of insulin insensitivity. When glucose tolerance is minimally impaired the plasma concentration of insulin is normal or frequently supranormal (Hales and Randle, 1963; Hales, Randle and Walker, 1964). There is moreover evidence that the rate of release of fatty acids is accelerated at this stage insofar as the plasma concentrations of fatty acids are augmented.
This has raised the interesting possibility tha an abnormality in the release of fatty acids the primary event in the development of diabetes leading secondarily to insensitivity towards the hypoglycæmic effect of insulin and to impaired glucose tolerance (Hales and Randle, 1963). Evidence for this hypothesis he been summarised in full elsewhere (Hales ang. Randle, 1963; Randle and others, 1963; Randle Garland, Hales and Newsholme, 1964). \& further point of evidence has recently been pros vided by the experimental observations made in man by Felber and Vannotti (1963) who have raised plasma NEFA by infusion of triglycerides. This led to impaired glucose tolerance and insulin insensitivity and the plasma concentrations of glucose and insulim during oral glucose tolerance tests were vero similar to those seen in early diabetes. The findings provide a striking demonstration i i man that an increased provision of fatty acids. for respiration can lead to impaired glucose tolerance and insulin insensitivity.

\section{REFERENCES}

CHANCE, B. (1959): Quantitative Aspects of the Control of Oxygen Utilisation. Ciba Foundation Symposin $\vec{B}$ on the Regulation of Cell Metabolism (eds. G. E. W. Wolstenholme and C. M. O'Connor). pp. 91- $\$ 2 \$$ London: J. \& A. Churchill.

Cornblath, M., Randle, P. J., Parmeggiani, A., and Morgan, H. E. (1963): Regulation of Glycogenolysist Muscle. Effects of Glucagon and Anoxia on Lactate Production, Glycogen Content and Phosphorytase Activity in the Perfused Isolated Rat Heart. J. biol. Chem., 238, 1592.

DruRY, D. R., and WICK, A. N. (1953): Can Other Fuels Substitute For Glucose in Tissues Subjected to Inten Insulin Activity? Ciba Foundation Colloquia Endocrin., 6, 211.

Felber, J. B., and VANNOTTI, A. (1964): Effects of Fat Infusion on Glucose Tolerance and Insulin Plasma Levels. Med. Exp. (Basel), 553, 1.

Garland, P. B., Newsholme, E. A., and Randle, P. J. (1962): Effects of Fatty Acids, Ketone Bodies, Diabet $\overrightarrow{\bar{E}}$ and Starvation on Pyruvate Metabolism in Rat Heart and Diaphragm Muscles. Nature, (Lond.), 195, 38.

GARLAND, P. B., and RANDLE, P. J. (1963): Effects of Alloxan-Diabetes and Adrenaline on Conoentrations of Free Fatty Acids in Rat Heart and Diaphragm Muscles. Nature, (Lond.), 199, 381.

Garland, P. B., Randle, P. J., and NewSholme, E. A. (1963): Citrate as an Intermediary in the Inhibition of Phosphofructokinase in Rat Heart Muscle by Fatty Acids, Ketone Bodies, Pyruvate, Diabetes and Starvat. tion, Nature, (Lond.), 200, 169. GARLAND, P. B., and RANDLE, P. J. (1964): Control of Pyruvate Dehydrogenase in the Perfused Rat Heart bo
the Intracellular Concentration of Acetyl-Coenzyme A. Biochem. J., 91, 6C.

HaLes, C. N., and RANDLE, P. J. (1963): Effects of Low Carbohydrate Diet and Diabetes Mellitus on Plasma Concentrations of Glucose, Non-esterified Fatty Acids and Insulin During Oral Glucose Tolerance Test Lancet, i, 790.

Hales, C. N., RANDle, P. J., and Walker, J. M. (19ß4): $\beta$-cell Function in Human Diabetes. In the Structu敦 and Metabolism of the Pancreatic Islets. Eds. S. Brolin, B. Hellman and H. Knutson. London: Pep
gamon Press.

LARNER, J. (1964): Insulin and the Control of UDPG- $\alpha$-glucan Transglucosylase Activity. In Ciba Foundatio Colloquium on Control of Glycogen Metabolism. Eds. G. E. W. Wolstenholme, M. P. Cameron and MonOD, J., CHANGEUX, J. P., and JACOB, F. (1963): Allosteric Proteins and Cellular Control Systems, J. mole
Biol., 6, 306.

Morgan, H. E., RANDLE, P. J., and REGEN, D. M. (1959): Regulation of Glucose Uptake by Muscle. 3. The Effects of Insulin, Anoxia, Salicylate and 2:4-dinitrophenol on Membrane Transport and Intracellular Phosphorylation of Glucose in the Isolated Rat Heart. Biochem. J., 73, 573 .

NewSHOLME, E. A., and RANDLE, P. J. (1961): Regulation of Glucose Uptake by Muscle. 5. Effects of Anoxi Insulin, Adrenaline and Prolonged Starving on Concentrations of Hexose Phosphates in Isolated Rat Dią, phragm and Perfused Isolated Rat Heart. Biochem. J., 80, 655.

NewSHOLME, E. A., RANDLE, P. J., and MANCHESTER, K., L. (1962): Inhibition of Phosphofructokinase RE 193, 270.
19erfused Rat Heart by Respiration of Ketone Bodies, Fatty Acids and Pyruvate. Nature, (Lond d, $_{3}$ 
Parmeggiani, A., and Morgan, H. E. (1962): Effects of Adenine Nucleotides and Inorganic Phosphate on Muscle Phosphorylase Activity. Biochem. biophys. Res. Commun., 9, 252.

Parmeggiani, A., and Bowman, R. E. (1963): Biochem. biophys. Res. Commun., 12, 268.

Passonneau, J. V., and LOWry, O. H. (1962): Phosphofructokinase and the Pasteur effect. Biochem. biophys. Res. Commun., 7, 10.

RANDLE, P. J. (1956): Anærobic Uptake of Glucose in vitro by the Isolated Rat Diaphragm. Nature, (Lond.), 178, 983.

RANDLE, P. J., and SMITH, G. H. (1958a): Regulation of Glucose Uptake by Muscle. 1. The Effects of Insulin, Anærobiosis and Cell Poisons on the Uptake of Glucose and Release of Potassium by Isolated Rat Diaphragm. Biochem. J., 70, 490.

RanDle, P. J., and SMith, G. H. (1958b): Regulation of Glucose Uptake by Muscle. 2. The Effects of Insulin, Anærobiosis and Cell Poisons on the Penetration of Isolated Rat Diaphragm by Sugars. Biochem. J., 70, 501 .

Randle, P. J., Garland, P. B., Hales, C. N., and Newsholme, E. A. (1963): The Glucose Fatty Acid of Cycle. Lancet, 1, 785 .

Randle, P. J., Garland, P. B., Hales, C. N., and Newsholme, E. A. (1964): The Glucose Fatty Acid Cycle and Diabetes Mellitus. Ciba Foundation Colloquia Endocrin., 15, 192.

ShipP, J. C., OPIE, L. H., and Challoner, D. R. (1961): Fatty Acid and Glucose Metabolism in the Perfused Heart. Nature, (Lond.), 189, 1018.

Sutherland, E. W., and RALl, T. W. (1960): The Relation of Adenosine 3', 5'-monophosphate and Phosphorylase to the Actions of Catecholamines and Other Hormones. Pharmacol. Rev., 12, 1 .

Tata, J. R., ERnSter, L., Lindberg, O., Arrhenius, E., Pedersen, S., and Hedman, R.: The Action of Thyroid Hormones at the Cell Level. Biochem. J., 86, 408.

Tiederman, H., and BORN, J. Z. (1959): Versuche zum Mechanisms der Pasteur-Reaktion Der Ein fluss von Phosphationen auf die Activitat der strukturgebunden Hexokinase. Z. Naturforsch., 14b, 477.

Vaughan, M., Berger, J. E., and Steinberg. D. (1964): Hormone Sensitive Lipase and Monoglyceride Lipase Activities in Adipose Tissue. J. biol. Chem., 239, 401.

VINUElA, E., SAlAS, M., and SOLS, A. (1963): Glucokinase and Hexokinase in Liver in Relation to Glycogen Synthesis. J. biol. Chem., 238, 1175.

WALKER, D. G., and RAO, S. (1964): The Role of Glucokinase in the Phosphorylation of Glucose by Rat Liver. Biochem. J., 90, 360.

Williamson, J., R., and KReBS, H. A. (1961): Acetoacetate as Fuel of Respiration in the Perfused Rat Heart. Biochem. J., 80, 540. 